CAHIERS DE

NARRATOLOGIE

\section{Cahiers de Narratologie}

Analyse et théorie narratives

37 | 2020

Approches transmédiales du récit dans les fictions contemporaines

\title{
Approches transmédiales du récit dans les fictions contemporaines
}

Rémi Cayatte et Anaïs Goudmand

\section{(2) OpenEdition}

Journals

Édition électronique

URL : http://journals.openedition.org/narratologie/10392

DOI : 10.4000/narratologie. 10392

ISSN : 1765-307X

Éditeur

LIRCES

Référence électronique

Rémi Cayatte et Anaïs Goudmand, « Approches transmédiales du récit dans les fictions

contemporaines », Cahiers de Narratologie [En ligne], 37 | 2020, mis en ligne le 04 septembre 2020,

consulté le 10 décembre 2020. URL : http://journals.openedition.org/narratologie/10392 ; DOI :

https://doi.org/10.4000/narratologie.10392

Ce document a été généré automatiquement le 10 décembre 2020.

Article L.111-1 du Code de la propriété intellectuelle. 


\title{
Approches transmédiales du récit dans les fictions contemporaines
}

\author{
Rémi Cayatte et Anaïs Goudmand
}

Ce numéro des Cahiers de Narratologie réunit des articles issus d'une partie des interventions qui ont eu lieu lors des premières Assises de la recherche en cultures populaires et médiatiques, organisées à l'initiative de l'Association internationale des chercheurs en littératures populaires et culture médiatique, du 11 au 13 octobre 2018 à l'Université Paris Nanterre et à l'Université Paris Sorbonne. Les travaux qui composent ce dossier ont pour objectif commun d'aborder les fictions non pas à l'échelle d'un seul média, mais à l'échelle de l'écologie médiatique au sein de laquelle elles s'inscrivent. Depuis plusieurs décennies, les théories du récit tendent à s'affranchir des approches monomédiales, pour aborder la culture médiatique de manière plus large, ce qui implique un renouvellement des outils et des méthodes auquel les articles de ce dossier contribuent.

2 Si la médialité se définit comme la manière dont un récit est conditionné par le média au sein duquel il prend forme (Ryan 2012: \$3), la transmédialité, quant à elle, " concerne les phénomènes qui ne sont pas spécifiques à un média individuel et/ou qui sont examinés dans le cadre d'une analyse comparative des médias qui ne se concentre pas sur un média source particulier » (Wolf 2011:5, n. t.). Les approches transmédiales du récit ont permis, dans un premier temps, d'élargir l'analyse narratologique à différents supports sémiotiques et technologiques. Des concepts nativement littéraires ont ainsi pu être enrichis, complétés ou amendés, comme cela a été le cas dans l'analyse filmique proposée par Seymour Chatman (1978), ou encore par François Jost (1987), qui a par exemple substitué à la focalisation genettienne le terme d'ocularisation, plus à même d'envisager les effets de monstration cinématographique. Dans en second temps, elles ont cherché à se détacher plus nettement de l'ancrage littéraire de la narratologie et à s'intéresser aux fictions qui déploient leur(s) récit(s) à travers différents supports faisant partie des objets culturels les plus largement consommés actuellement (Cayatte 2018). Le gain heuristique d'une approche comparative des reformulations de récits et d'univers de fiction dans différents supports s'avère double, puisqu'elle permet à la fois 
de prendre en compte leurs nouveaux usages, mais aussi, en retour, d'affiner la compréhension générale de la narrativité, ainsi que le formulent Jan-Noël Thon ou encore Raphaël Baroni :

Si l'on reconnaît qu'une partie significative de la culture médiatique contemporaine est définie par des représentations narratives, et si l'on accepte que l'examen de leurs similitudes ainsi que de leurs différences peut aider à expliquer [...] les adaptations intermédiales et les franchises de divertissement [...] tout en contribuant à une meilleure compréhension générale des formes et des fonctions des productions narratives à travers les médias, il devient évident que les études médiatiques ont besoin d'une véritable narratologie transmédiale. (Thon 2016: XVIII, n.t.)

[E]n travaillant sur la transférabilité des concepts narratologiques à différents médias nous enrichirons non seulement la compréhension de la manière dont chaque support conditionne la représentation narrative, mais également, sur une échelle élargie, la connaissance portant sur ce que tous les récits, quelle que soit la substance qui les incarne, ont en commun. (Baroni 2017 : 174)

3 Avant de présenter les différents textes qui composent ce dossier consacré aux approches transmédiales du récit dans les fictions contemporaines de manière plus détaillée, il convient de se livrer à un éclaircissement terminologique. Les articles ici rassemblés ne portent pas nécessairement sur des ensembles transmédiatiques qui mobilisent en convergence des dispositifs sémiotico-techniques variés. Dans les termes désormais incontournables d'Henry Jenkins, le «storytelling transmédia » est défini comme " un processus à travers lequel les éléments d'une fiction sont dispersés sur plusieurs plateformes médiatiques dans le but de créer une expérience de divertissement coordonnée et unifiée » (2007: \$2, n. t.). Outre les critiques que l'on pourrait adresser à une telle notion, qui tend à généraliser abusivement le modèle d'une production transmédiatique d'emblée maîtrisée et coordonnée (Ryan 2017), les problématiques soulevées par la transmédialité ne se réduisent pas à ce mode de narration caractéristique de la culture de la convergence. Il convient donc de distinguer nettement entre les approches transmédiales du récit, et les objets sur lesquels elles portent, ces derniers n'étant pas nécessairement transmédiatiques (Baroni \& Goudmand 2019). Les articles de ce dossiers, tout en adoptant des perspectives différentes (diachroniques, historiques, économiques, conceptuelles), s'inscrivent dans cette approche transmédiale plus que transmédiatique, en abordant différentes comparaisons entre des objets médiatiquement hétérogènes pour en faire ressortir les multiples points de contact sur le plan narratologique.

4 En ouverture de ce dossier, Matthieu Letourneux adopte, dans la lignée de ses travaux précédents, une perspective historique et communicationnelle: il montre que les questions d'ordre esthétique et poétique ne sont pas détachées des problématiques économiques et industrielles. Il s'intéresse ici plus spécifiquement aux fictions produites ces vingt-cinq dernières années dans le cadre du régime postfordiste, qui se distingue notamment du régime fordiste par des stratégies de singularisation: elles combinent désormais des niveaux de lecture variés et ne s'adressent ainsi plus à un public spécifique mais à des publics différenciés. La variété des réappropriations faniques, critiques, contre-culturelles, etc. est en quelque sorte structurellement inscrite dans le système de production des fictions postfordistes. Il aborde donc la question de la sérialité à un niveau pré-narratif, celui des logiques de production, et souligne l'importance de la notion de marque : lorsque la mise en récit des différentes extensions des fictions sérielles obéit par exemple à un principe d'unification 
diégétique, il s'agit avant tout d'une stratégie entrepreneuriale. En creux, c'est un programme pour la narratologie qui se dessine: dans le contexte des productions postfordistes, l'analyse des effets narratifs ne peut avoir lieu que secondairement et doit être systématiquement saisie comme un symptôme, historiquement, économiquement et médiatiquement situé, d'une logique qui l'englobe, celle de la construction d'une marque. La dynamique de monde fictionnel et la dynamique de marque fonctionnent ainsi comme les deux faces d'une même pièce qu'il serait impropre de désolidariser.

5 Les interrogations soulevées par Bounthavy Suvilay dans le deuxième article de ce dossier s'inscrivent dans la même perspective historique, tout en sortant du périmètre le plus volontiers exploré par les chercheurs européens et nord-américains : son article est consacré à la culture médiatique japonaise, dans laquelle les prolongements transmédiatiques et transfictionnels des fictions sources sont pris dans une logique sérielle. Elle développe une approche diachronique attentive aux logiques de production spécifiques au contexte japonais, marqué par des alliances multisectorielles entre éditeurs de mangas, chaînes de télévision, éditeurs de jeux vidéo et sponsors. Elle montre notamment que les industries du papier perdent progressivement leur position centrale au profit de celles de la télévision ou du jeu. Ainsi, jusque dans les années 1990, les partenariats se développent autour du manga, dont les droits sont détenus par l'auteur. Le récit devient dès lors un outil de marketing qui attire des consommateurs pour les jeux et jouets, et se déploie à travers les différents supports sans véritable cohérence interne, sur le modèle de Dragon Ball Z. Les logiques narratives évoluent ensuite vers des logiques de collection, ce qui est directement lié aux nouveaux usages : la consommation du jeu vidéo précède désormais celle des mangas, suivant le modèle de Pokémon. Son étude s'achève sur la mise au jour d'une troisième phase, qui correspond à l'essor du numérique : au sein des adaptations transmédiatiques le manga n'est plus qu'une étape optionnelle, sur le modèle de Sword Art Online. Elle montre ainsi qu'en dépit de l'autonomisation croissante du personnage, le récit ne disparaît pas pour autant: les dynamiques narratives se déploient sous d'autres formes, ce qui implique d'orienter les modèles d'analyse du récit dans de nouvelles directions.

6 Maxime Prévost cherche quant à lui à démontrer la manière dont une notion construite par un individu parvient à s'installer durablement dans l'imaginaire collectif, fondant une mythologie moderne et laïque. S'appuyant sur une perspective mythocritique, il aboutit à une reconfiguration des notions de mythologie et de mythe à l'aune de la culture médiatique. Il s'intéresse plus précisément aux sources du devenir social et collectif du "monde perdu», qui a connu, depuis Jules Verne, de nombreuses déclinaisons, notamment cinématographiques, et qui demeure toujours vivace de nos jours. Le «monde perdu » se caractérise par sa capacité à proposer des réponses à des questions collectives qui émergent dans l'histoire de sa réception: il permet notamment de cristalliser des interrogations liées à la disparition d'un lien exploratoire et aventurier à la nature. Cette topique devient ainsi, au fil du temps, une mythologie. La notion de "vecteur mémoriel » lui permet d'éclairer ce qui assure au mythe sa persistance collective et son renouvellement transmédiatique, qui procède par successions d'adaptations se superposant à l'œuvre initiale sans pour autant l'oblitérer (comme dans le cas différentes incarnations de Sherlock Holmes au cinéma et à la télévision par exemple). 
7 L'enjeu de décloisonnement de l'analyse des fictions médiatiques sur lequel ce dossier repose est au cœur de l'article de Simon Bréan qui, s'appuyant sur des récits de sciencefiction, montre que l'étude de médias variés permet d'enrichir la compréhension de la littérature. Il s'intéresse au cas des artefact fictionnels, c'est-à-dire des objets pseudodocumentaires qui peuplent les mondes fictionnels (articles de journaux, d'encyclopédies, clips de vidéosurveillance...). Son hypothèse est que ces artefacts fictionnels mettent en jeu un mécanisme cognitif similaire au-delà des différences de support. Selon lui, la métaphore de l'enquêteur généralement privilégiée pour décrire la posture du récepteur s'avère d'une part peu pertinente pour les autres médias que la littérature, d'autre part limitée pour décrire la situation du lecteur face aux artefacts d'un roman de science-fiction. Il propose de lui substituer un modèle d'immersion ludique, celui du joueur de jeu de rôle. On assiste ainsi a l'inversion du paradigme comparatiste auparavant majoritaire en théorie du récit: il ne s'agit plus d'emprunter des notions nativement littéraires pour les adapter à d'autres médias, en tenant compte de leurs spécificités, mais d'évaluer dans quelle mesure des concepts issus par exemple de la ludologie peuvent s'avérer fructueux pour la littérature ou le cinéma. Les implications des réflexions de Simon Bréan débordent le cadre de la seule sciencefiction et débouchent sur une reconception de la notion d'immersion, qui est conçue non pas comme une expérience par défaut, mais comme un positionnement axiologique du récepteur face à une fiction donnée. La modélisation gagne ainsi en souplesse, et permet d'inclure des postures de réception différenciées.

8 Martin Ringot, à l'inverse de Simon Bréan, accorde dans son étude une place centrale au modèle du «narrataire-enquêteur». Il propose de placer la dynamique de coopération interprétative des récepteurs au centre de l'étude des formes de narration vidéoludique, et tout particulièrement dans le cas des jeux vidéo en monde ouvert. Adaptant la notion de worldness à ces supports de fiction, il propose d'étendre le concept de mondialité en y incorporant la prise en compte des processus techniques permettant la matérialisation à l'écran d'une diégèse, ainsi que des possibles narratifs en puissance, qui faute d'interaction de la part des récepteurs, ne sont pas toujours réalisés ou réalisables. En abordant la question du rôle des joueurs dans les fictions interactives, cet article propose de considérer ces derniers comme des narratairesenquêteurs, qui agencent et relient, de manière pragmatique tout autant qu'interprétative et narrative, les informations glanées au fil de l'exploration d'univers fictionnels. De cette dynamique d'agencement par les récepteurs découle à la fois une multiplicité de mondes possibles, mais également un rôle moins central accordé au récit dans les supports vidéoludiques de la fiction que dans d'autres dispositifs, ce que l'auteur propose de considérer comme une inversion de la hiérarchie établie entre les deux pôles du récit et de la narration. Le texte aboutit à une proposition de rejet de l'idée de co-construction par les joueurs des récits vidéoludiques, l'empirisme de ces récits davantage spatialisés que temporels aboutissant à la coexistence de multiples trajectoires narratives plus qu'à leur co-construction.

9 La question du réagencement est également au cœur de l'article de Frédéric Aubrun et Vladimir Lifschutz, consacré à l'analyse des processus d'adaptation des certains récits super-héroïques. Leur travail sur les origin stories de super-héros est construit sur une prise en compte des fondements fictionnels, formels et contextuels du processus d'énonciation des comic books. Ils mettent en évidence des invariants à la fois dans la figure du super-héros et dans la morphologie des récits de leurs origines et s'appuient 
sur les outils fournis par le schéma actanciel de Greimas et les sphères d'action pour étudier l'adaptation des origins stories de super-héros dans des séries télévisées contemporaine. En envisageant plus particulièrement la dimension sociétale des réécritures contemporaines opérées par les récentes séries Gotham et Watchmen, cet article démontre que les adaptations de ces récits sont des formes d'actualisation thématique et contextuelle qu'il est possible d'éclairer par l'analyse de leurs évolutions narratives et formelles, transformant à la fois le récit super-héroïque en tant que genre et en tant que processus de communication.

En clôture de ce dossier, Fanny Barnabé aborde une forme d'adaptation transmédiatique particulière: les machinimas. Ces productions audiovisuelles sont composées de captures de sessions de jeu vidéo qui sont utilisées pour produire des séquences s'apparentant à des films d'animation, au croisement du théâtre de marionnettes et des techniques de stop motion. Cet article fait ressortir différentes figures rhétoriques prototypiques de ces productions dans le but d'en souligner les particularités discursives et narratives. Ces figures se caractérisent principalement par leurs dynamiques d'écart, de polyphonie et de disjonction, ce qui est autant lié au contexte pragmatique de production des machinimas qu'à l'hybridité centrale de ces dispositifs. Cette hybridité intrinsèque génère volontiers des formes de mise en abyme et de métalepse, ce que Fanny Barnabé souligne dans son analyse des reformulations du dessin animé Pokémon dans les deux machinimas Garrymon et Halomon, qui remobilisent respectivement des matériaux audiovisuels et des références ludiques tirées des jeux Garry's Mod et Halo: Reach. Entre rupture et indexicalité, les formes de narration sous contraintes produites par les machinéastes proposent une forme particulière de narration (par le) ludique, autant à travers la réappropriation de moteurs et de séquences de jeu qu'à travers la réappropriation des fictions qu'ils mettent en scène de manière créative et réflexive.

11 Les articles de ce dossier ont en commun d'aborder, chacun à leur manière, les récits médiatiques sous l'angle des trajectoires narratives: ils soulignent que le récit n'est pas un objet figé, mais un processus vivant et vivace, qu'il s'agisse des cheminements de leur production ou de leur réception, qui se construisent à travers une succession de reformulations et de réappropriations. On le voit, les pistes explorées pour illustrer cette idée sont diverses. Certains articles défendent une approche historiquement contextualisée des fictions médiatiques (Letourneux, Suvilay), qui permet de saisir l'évolution des pratiques narratives en la mettant en lien avec celle des stratégies industrielles qui dictent la mise en forme des objets et avec celle des usages des publics. Pour les autres, l'étude d'objets issus de la culture médiatique appuie la recherche d'invariants de la narrativité, en d'autres termes, de mécanismes narratifs communs aux différents supports sur lesquels peuvent se déployer les récits. L'ensemble de ce dossier contribue ainsi à alimenter une réflexion transmédiale : il s'agit de développer des outils et des méthodes à même d'interroger les effets de circulation entre les médias (qui sont particulièrement saillants dans le contexte de la culture médiatique), sans pour autant effacer les spécificités des différents supports sémio-techniques et de leurs usages. 


\section{BIBLIOGRAPHIE}

Baroni, Raphaël (2017), « Pour une narratologie transmédiale », Poétique 2017/2, n 182, p. $155-175$.

Baroni, Raphaël \& Anaïs Goudmand (2019) « Narratologie transmédiale / Transmedial Narratology », Glossaire du RéNaF. En ligne, consulté le 6 mai 2020. URL : https://wp.unil.ch/ narratologie/2019/03/narratologie-transmediale-transmedial-narratology/

Cayatte, Rémi (2018) « Temps de la chose-racontée et temps du récit vidéoludique : comment le jeu vidéo raconte? », Sciences du jeu, 9|2018. En ligne, consulté le 6 mai 2020. URL : https:// journals.openedition.org/sdj/936

Chatman, Seymour (1978), Story and Discourse : Narrative Structure in Fiction and Film, Ithaca (N. Y.), Cornell University Press.

Jenkins, Henry (2013 [2006]), La culture de la convergence. Des médias au transmédia, Paris, Armand Colin.

Jenkins, Henry (2007), « Transmedia Storytelling 101 », Confessions of an Aca-fan. En ligne, consulté le 6 mai 2020. URL : http://henryjenkins.org/blog/2007/03/transmedia_storytelling_101.html Jost, François (1987), L'œil-caméra. Entre film et roman, Lyon, Presses Universitaires de Lyon, «Linguistique et sémiologie ».

Ryan, Marie-Laure (2012), « Narration in various media », The Living Handbook of Narratology. En ligne, consulté le 6 mai 2020. URL : https://www.lhn.uni-hamburg.de/article/narration-variousmedia

Ryan, Marie-Laure (2017), « Le transmedia storytelling comme pratique narrative », Revue française des sciences de l'information et de la communication, 10|2017. En ligne, consulté le 6 mai 2020. URL : http://journals.openedition.org/rfsic/2548

Thon, Jan-Noël (2016), Transmedial Narratology and Contemporary Culture, Lincoln, University of Nebraska Press.

Wolf, Werner (2011), « (Inter)mediality and the Study of Literature », Comparative Literature and Culture, $n^{\circ} 13$ (3). En ligne, consulté le 6 mai 2019. URL : https://docs.lib.purdue.edu/clcweb/ vol13/iss $3 / 2 /$

\section{AUTEURS}

\section{RÉMI CAYATTE}

CERTOP, Université Toulouse III Paul Sabatier.

Rémi Cayatte est maître de conférence en sciences de l'information et de la communication à l'université Toulouse III. Ses travaux portent sur les spécificités narratives et persuasives des formes interactives de communication et de médiation. Il travaille notamment dans ce cadre sur les dispositifs de jeu (vidéo), en croisant les outils et apports des sciences du jeu et des sciences de l'information et de la communication. 


\section{ANAIIS GOUDMAND}

Anaïs Goudmand est postdoctorante à la KU Leuven. Sa thèse, intitulée Récits en partage. Expériences de la sérialité narrative en culture médiatique, a été dirigée par Raphaël Baroni (Université de Lausanne) et Jean-Marie-Schaeffer (EHESS). Elle porte sur les différentes formes que revêt l'expérience des récits sériels, telles qu'elles sont modelées par les contraintes de production et de diffusion de la culture médiatique. Trésorière de la LPCM (association des chercheurs en Littérature Populaire et Culture Médiatique), elle est co-administratrice du site WordPress du Réseau des narratologues francophones. Elle fait partie des membres fondateurs du Séminaire Littéraire des Armes de la Critique et elle est membre du comité de lecture de la revue en ligne Proteus - Cahiers des théories de l'art. 